\title{
A EFICÁCIA DA NORMA ISO 362:2007 PARA O CONTROLE DO RUÍDO DE VEÍCULOS PESADOS
}

\author{
R. de Abrantes ${ }^{1}$; A. L. S. Forcetto ${ }^{2}$; R. M. Araújo³ \\ ${ }^{1}$ Companhia Ambiental do Estado de São Paulo, Doutor em Saúde Pública \\ 2 Companhia Ambiental do Estado de São Paulo, Mestrando em Ambiente, Saúde e \\ Sustentabilidade \\ ${ }^{3}$ Companhia Ambiental do Estado de São Paulo, Tecnólogo mecânico \\ rabrantes@sp.gov.br; aforcetto@sp.gov.br; renaraujo@sp.gov.br
}

\section{RESUMO}

A Europa está mudando sua regulamentação sobre ruído de veículos automotores, pois perceberam que o ruído urbano tem sido maior do que o esperado devido ao crescimento da frota de veículos e a ineficiência do método de homologação atual. Em resposta a esse problema, o método de avaliação do ruído de passagem foi revisado e atualmente os veículos estão sendo testados sob condições mais realistas.

Existem alguns pontos críticos nesse novo método que tendem enfraquece-lo, por exemplo, a penalização do controle do ruído dos pneus, a desconsideração do ruído do ventilador do sistema de arrefecimento e a avaliação de ônibus e caminhões sob as mesmas circunstâncias.

O objetivo deste artigo é discutir o novo método de avaliação de ruído de passagem para veículos pesados e identificar pontos de melhoria para que regulamentação seja mais efetiva e melhor aceita por outros países que não pertençam à União Europeia.

Palavras-chaves: ruído veicular, poluição sonora, ISO 362-1, ruído de passagem.

\section{INTRODUÇÃO}

A norma internacional sobre ruído veicular - ISO 362-1 - recebeu uma substancial modificação em 2007 e algumas alterações menores em 2009 e 2015 e a partir de 2016 será o procedimento em vigor para a União Europeia homologar os veículos leves de passageiros, caminhões e ônibus, como definido no Regulamento 540/2014 do Parlamento Europeu [1]. Os pressupostos para estas atualizações são de ter um procedimento mais próximo do comportamento em rua dos motoristas, sendo então mais realista e, assim, mais eficaz no controle do ruído veicular.

Este artigo tem como objetivo discutir a abordagem dada pela norma ISO $362-$ 1:2007 para os veículos pesados (VP) nas categorias M2, M3, N2 e N3 (ver anexo), 
i.e., basicamente ônibus e caminhões, para identificar pontos possíveis de melhoria. O foco nos VP se dá porque estas categorias tipicamente possuem nível de ruído maior do que os veículos leves de passageiros (VLP) e porque permanecem alguns aspectos não considerados pela norma que podem fazer com que os resultados globais não sejam tão eficazes como esperado. VLP serão discutidos também, mas somente com o propósito de compará-los com os VP. Qualidade de pista, pneus e veículos da categoria $L$ (motocicletas e veículos de três rodas), cujas participações na emissão de ruído são também muito importantes, não são considerados neste artigo.

\section{PORQUE CONTROLAR O RUÍDO VEICULAR}

O ruído nos centros urbanos é devido a diferentes fontes, como sirenes, aeronaves, ferrovias e tráfego rodoviário, entre outros [2]. Contudo, através de consultas entre moradores de algumas cidades europeias, concluiu-se que a perturbação por barulho se deve principalmente aos veículos ao longo das ruas principais e durante transientes de aceleração [3].

Há forte correlação entre o ruído e doenças cardiovasculares, deterioração do comprometimento cognitivo, sensação de zumbido e perturbação do sono. Em populações expostas a altos níveis de ruído há correlação exponencial para pressão alta e infarto do miocárdio. A perturbação do sono está relacionada com uma longa lista de problemas, afetando a coordenação motora, a consolidação da memória, criatividade, levando a adoção de comportamentos de risco, falha em desempenho de detecção de sinais e risco de acidentes. As pessoas sujeitas a elevados níveis de ruído, mesmo ruídos intermitentes, especialmente durante a noite podem não perceber a razão de distúrbios do sono, mas podem sentir os efeitos [3].

O ruído nos centros urbanos está acima do nível recomendado pela OMS em muitos países ao redor do mundo, sendo que o ruído do tráfego rodoviário é classificado como o segundo principal estressor ambiental urbano, resultando em distúrbios de saúde. Um agravante é que a poluição por emissão de gases nocivos vem se reduzindo ao longo dos anos, mas a poluição sonora tem aumentado progressivamente [3].

No processo de desenvolvimento de um veículo, os fabricantes devem garantir o cumprimento da legislação, o que tem de ser verificado antes do lançamento do modelo no mercado. Na Europa, houve reduções graduais do limite de ruído permitido para a homologação de veículos ao longo do tempo. Para os VP, por exemplo, o limite diminuiu de $91 \mathrm{~dB}(\mathrm{~A})$ na década de setenta para $80 \mathrm{~dB}(\mathrm{~A})$ em 1997. Isto significa que o ruído nos centros urbanos deveria ter reduzido pela renovação da frota ao longo do tempo ou que seria possível multiplicar por dez a frota de veículos da época para se obter o mesmo nível de ruído nas ruas. Mas em termos práticos, a redução foi muito inferior ao esperado [3] e [4]. SANDBERG aponta que, devido ao aumento do fluxo de veículos e da velocidade média, o Leq na Alemanha entre 1975 e 1992 teve um aumento de 2,5 dB(A) em vias expressas [5]. 
Em veículos, as causas da geração de ruído e as subsequentes características de irradiação da fonte de ruído são, em geral, muito complexas. A principal irradiação de ruído do motor ocorre atrás do eixo dianteiro através do espaço entre o lado inferior da carroçaria do veículo e o solo em direção às pessoas [6]. Em experimentos de isolamento de fontes de ruído em veículos utilizando a técnica de blindagem, a fim de identificar suas características, observou-se que o nível relativo de cada fonte de ruído depende da posição do veículo na pista de ensaio em relação ao observador. Assim, no teste de ruído padronizado conforme a norma ISO 362-1, no início da pista de ensaio o ruído do sistema de ar de admissão é dominante. No meio da pista, quando a distância do veículo em relação ao microfone é menor, o ruído mais elevado é do conjunto propulsor. No final da pista, o ruído do sistema de exaustão é dominante [6].

\section{MÉTODO DE CONTROLE ATUAL - ISO 362:1998}

$\mathrm{Na}$ Europa, a regulamentação de ruído em veículos utilizada até então, Diretiva $70 / 157 / C E$, foi baseada na norma ISO 362:1998, que determina os procedimentos para execução do teste de ruído de passagem ou em aceleração e será denominada como método A neste artigo, a fim de facilitar a leitura e entendimento [7].

A instalação de ensaio (pista) é composta por uma área de aceleração quadrada de 20 metros de lado revestida de asfalto, que segue os requisitos da norma ISO 10844, uma vez que a qualidade da superfície pode interferir nos resultados. Há uma linha de referência ao longo de seu centro, representando o sentido de condução do veículo.

Dois microfones são posicionados a meio caminho na área de aceleração, a uma distância de 7,5 m de cada lado da linha de referência e a uma altura de 1,2 metros do solo. Os testes em VLP são feitos da seguinte forma: o veículo é conduzido na linha central de referência, aproximando-se da área de aceleração em velocidade constante de $50 \mathrm{~km} / \mathrm{h} \pm 1 \mathrm{~km} / \mathrm{h}$. Quando a parte dianteira do veículo atingir o limite inicial da área de aceleração, este é totalmente acelerado e o veículo passa pela área de medição na condição de aceleração total do veículo (em inglês: Wide Open Throttle - WOT). Quando a traseira do veículo deixa a área de teste, a avaliação se encerra. O nível de ruído máximo (pressão sonora em decibéis com escala ponderada $A$ ) da passagem é registrado pelos dois microfones. Para a maioria dos VLP com transmissões manuais, a passagem é realizada em $2^{\underline{a}}$ e $3^{\underline{a}}$ marchas. Quatro corridas consecutivas são realizadas para cada marcha. O resultado final é dado pela média de todas as passagens válidas.

Durante o teste, os dados meteorológicos devem estar dentro de certos limites de tolerância, e devem ser monitorados. Altas velocidades de vento (acima de $5 \mathrm{~m} / \mathrm{s}$ ) e chuva impedem o teste.

O único critério de decisão do teste do ruído de passagem é o nível máximo de pressão sonora. A qualidade ou a frequência do som não são consideradas. Os níveis de ruído de passagem medidos em cada marcha devem estar dentro de um 
intervalo de $2 \mathrm{~dB}(\mathrm{~A})$, caso viole este limite, o resultado da passagem é descartado [7], [8].

Para os VP a pista de ensaio é a mesma, sendo que a principal diferença está relacionada com a velocidade de aproximação. A velocidade alvo corresponde a 3/4 da rotação de potência máxima para veículos equipados com motores abaixo de 225 kW ou a metade da rotação de potência máxima para os veículos equipados com motores superiores a $225 \mathrm{~kW}$ [7], [8]. Da mesma forma como nos VLP, os resultados devem estar dentro de um intervalo de $2 \mathrm{~dB}(\mathrm{~A})$ para serem considerados válidos.

\section{POR QUE UM NOVO MÉTODO}

Uma das principais críticas em relação a eficácia do método $A$ é a simulação inadequada do uso urbano do VLP, pela aceleração máxima (WOT) em segunda e terceira marchas. Devido ao WOT, o teste de passagem atual destaca o ruído do sistema motriz do veículo, em especial a admissão e o escape, que diminui a contribuição relativa do ruído do pneu. Isso foi agravado por mudanças na ISO 362:1998 que permitiram o uso de pneus com profundidade mínima dos sulcos. Como o comportamento típico urbano consiste em um misto de aceleração parcial e de trafegar em velocidade constante, há maior contribuição dos pneus no ruído emitido pelo veículo nas ruas [9], [10].

Outra razão para a simulação inadequada de ruído típico urbano é o desenvolvimento da tecnologia dos motores e das transmissões automáticas, automatizadas e CVT que fez com que alguns dos pressupostos técnicos originais da ISO 362:1998 não fossem mais válidos [10].

Assim, considerando-se a baixa eficiência do método $A$, a Comunidade Europeia propôs um novo procedimento com o intuito de obter uma medição do nível de ruído de veículos mais próxima do que se gera em trânsito urbano e que seja mais adequado para as novas tecnologias de propulsão e de transmissão. Sua proposta é ser mais eficaz na redução da emissão de ruído do veículo a partir de uma nova métrica, que é denominado como método $B$ neste artigo, a fim de facilitar a leitura [10].

\section{4 - O NOVO MÉTODO DE CONTROLE - ISO 362:2007}

A fim de entender melhor o uso de veículos em ambientes urbanos, foram coletados, em cidades europeias e japonesas, dados de 61 veículos em uso urbano real, de quase todas as categorias de veículos (M1, N1, N2, N3 e um de até 19 toneladas). Esta lista inclui veículos de potência variando de $40 \mathrm{~kW}$ a $440 \mathrm{~kW}$. Apesar de ter sido avaliado apenas um veículo da categoria N3 e nenhum ônibus, estas amostras foram consideradas representativas do mercado europeu [10].

$O$ procedimento definido pelo método $B$ mantém algumas semelhanças com $O$ método $A, p$. ex. utilizando as mesmas instalações de teste. Para VLP, os veículos são testados em duas condições: 
a) em aceleração, com velocidade alvo ainda de $50 \mathrm{~km} / \mathrm{h}$, mas deve ocorrer quando a parte dianteira do veículo atingir a linha central entre os dois microfones. A aceleração não é mais WOT, mas uma aceleração parcial, a fim de simular as condições de tráfego urbano, depende da relação entre a potência dos motores e massa dos veículos (PMR),

b) em velocidade constante a $50 \mathrm{~km} / \mathrm{h}$.

O resultado final para VLP é calculado por uma combinação ponderada dos resultados do ensaio de aceleração e os resultados do teste a velocidade constante, usando um fator de ponderação, de acordo com a relação entre potência do motor e a massa do veículo (em inglês: Power to Mass Ratio - PMR).

Para VP a aceleração típica urbana continua sendo o WOT, porém a velocidade alvo do veículo é de $35 \mathrm{~km} / \mathrm{h} \pm 5 \mathrm{~km} / \mathrm{h}$ e quando o ponto de referência do veículo alcançar o fim da área de aceleração, neste instante a rotação do motor deve estar entre $70 \%$ e $74 \%$ de rotação de torque máximo para veículos das categorias M2 acima de $3500 \mathrm{~kg}$ e N2. Para as categorias M3 e N3 a velocidade do motor deve estar entre $85 \%$ e $89 \%$ de rotação de torque máximo. O ponto de referência para VP é a extremidade frontal do veículo para veículos com motores na frente, ou a extremidade do motor mais próxima da frente do veículo para os demais. O ruído é medido a partir da linha de entrada até o ponto de referência ultrapassar cinco metros da linha de saída [1], [9] e [10].

Para limitar a aceleração dos veículos das categorias N2 e N3 em uma condição realista, é obrigatório adicionar carga no veículo, a uma proporção de $50 \mathrm{~kg} / \mathrm{kW}$, limitado a $75 \%$ do peso máximo permitido no eixo traseiro.

Este método baseia-se nos critérios de desempenho de aceleração e é independente da tecnologia de veículo, do tipo de transmissão, do número de engrenagens de transmissão e do tipo de motor. Esses critérios de desempenho tornam este método aplicável aos veículos atuais e futuros, incluindo transmissões adaptáveis automáticas, veículos híbridos, veículos elétricos e veículos a célula de combustível [10].

No Anexo A da norma ISO 362-1:2015 foram expostos os conceitos utilizados no desenvolvimento do novo procedimento para VLP e foram bem justificados, entretanto não houve justificativas para os VP, o que pode trazer dificuldade em ser amplamente aceito [4], [10].

\section{5 - ANÁLISE}

Há algumas razões que colocam em dúvida a eficácia do método $B$, tais como modificações em veículos usados, o controle da produção pode não ser eficaz, desconsideração do ruído do ventilador de arrefecimento, uso do mesmo método de homologação para aplicações diferentes, por exemplo, ônibus e caminhões. Isto faz com que o método B possa não ser adequado para homologar todos os tipos de veículos [11]. 
$A$ correlação entre o método $A$ e método $B$ é muito fraca para VLP, há uma tendência geral de os resultados dos testes feitos de acordo com o método $B$ serem um pouco inferiores [4]. Contudo, para VP nenhuma correlação entre os dois métodos foi encontrada na literatura. Por isso, ter limites de emissões menores necessariamente não significa obter benefícios ambientais, pois nas condições reais urbanas há a possibilidade de VP homologados pelo método $B$ emitir níveis sonoros mais elevados do que se tivessem sido homologados pelo método $A$.

Para VLP, o ruído de pneu é preponderante se comparado ao ruído da propulsão para as velocidades de 35 a $50 \mathrm{~km} / \mathrm{h}$ e se aplica especialmente a emissão a velocidades constantes. A contribuição da propulsão no ruído total de tráfego diminui com a velocidade, enquanto o ruído do pneu aumenta continuamente. Em velocidades superiores a $80 \mathrm{~km} / \mathrm{h}$ o ruído de propulsão em VLP é desprezível, contudo, no caso de VP a contribuição de ruído de propulsão não pode ser negligenciada, mesmo a velocidades mais elevadas [12].

Para atender ao método $\mathrm{B}$, a contribuição proporcional de ruído pelos pneus tornase maior, o que traz algumas dificuldades aos fabricantes de pneus, se considerado a tendência contraditória de se exigir pneus mais silenciosos e também "pneus verdes", i. e., pneus de baixa resistência ao rolamento [12]. Considerando-se que um isolamento da carroceria menos robusto será necessário, apesar de um provável aumento no custo dos pneus, espera-se diminuir o custo final dos veículos [13].

Em uma pesquisa sobre pneus para VP foram encontradas em ensaios padronizados de ruído diferenças entre eles de até $12 \mathrm{~dB}(\mathrm{~A})$, mostrando que pouco se sabe sobre os mecanismos que envolvem a geração de ruído para esta aplicação de pneus. Não está claro se será possível conceber pneus para pesados que produzam menos ruído durante o rolamento em estradas e, ao mesmo tempo, menor ruído do que os atuais pneus em condições urbanas, por isso a transferência de ônus dada aos fabricantes de pneus para VP pode não ser eficaz [12], [14] e [15]. Para ser capaz de desenvolver uma política mais abrangente para reduzir a emissão de ruído de pneus em VP, na prática, é necessário ter mais conhecimento sobre os fundamentos básicos dos mecanismos de emissão de ruído, os efeitos que as características dos pneus, as condições de funcionamento e do ambiente tem sobre a produção de ruído [15].

Há especialistas que consideram que os veículos têm quatro principais fontes de ruído: motor, sistema de admissão, sistema de escape e pneus, mas o ventilador de arrefecimento também deve ser considerado [6]. Esta importante fonte de ruído tem sido negligenciada de maneira preocupante por todos estes anos. O ventilador de arrefecimento pertence ao sistema de resfriamento, que atua junto ao radiador e pode ser acionado de modo permanente ou, eventualmente, por uma variedade de mecanismos como acoplamento viscoso, elétrico ou acionamento hidráulico. Resumidamente, a função principal do sistema de arrefecimento é a de manter a temperatura do motor dentro de uma faixa de trabalho adequada, geralmente cerca de $90^{\circ} \mathrm{C}$. Se a temperatura aumentar, o sistema de arrefecimento é acionado, evitando o superaquecimento e possível dano [16]. 
Nos VP o diâmetro do ventilador, não raramente, é maior do que meio metro e em pleno funcionamento pode aumentar o ruído dos veículos em até $6 \mathrm{~dB}(\mathrm{~A})$, o que corresponde a multiplicar por quatro a energia sonora liberada. É como se três outros veículos aparecem subitamente na rua, cada vez que o sistema de arrefecimento é ligado, por conseguinte, esta fonte de ruído não pode ser negligenciada.

Como citado, em ambos os métodos a medição do ruído emitido pelo veículo em movimento é considerada válida apenas quando a diferença entre os valores máximo e mínimo do mesmo lado do veículo não é maior do que $2 \mathrm{~dB}(\mathrm{~A})$ [8], [10]. Assim, quando a ventoinha de arrefecimento do sistema liga durante o teste, o ruído do veículo aumenta bem mais que os $2 \mathrm{~dB}(\mathrm{~A})$ e a leitura é desconsiderada. Em termos práticos, todas as vezes que o sistema de arrefecimento do motor funciona durante a avaliação de ruído, a leitura não é registrada. É como se o ruído do sistema de refrigeração não fosse significativo, ou como se as pessoas ou a biota não fossem afetadas por este tipo de ruído.

Uma avaliação nas ruas da região metropolitana de São Paulo (RMSP) foi realizada com ônibus urbanos em condições semelhantes ao método $A$ e observou-se que em 75 veículos avaliados, 42 estavam com o sistema de refrigeração desligado e que 0 ruído máximo (Lmáx) médio foi de 80,6 dB(A), enquanto 33 estavam com o sistema de refrigeração ligado e que o Lmáx médio foi de $84,8 \mathrm{~dB}(\mathrm{~A})$, ou seja, $4,2 \mathrm{~dB}(\mathrm{~A})$ acima da média do primeiro grupo de veículos. Noventa e quatro por cento dos ônibus que estavam com o sistema de arrefecimento funcionando excederam os limites de ruído de $81 \mathrm{~dB}(\mathrm{~A})$ para esta categoria de veículos (Figura 1).

Figura 1: Avaliação de ruído na RMSP, com 75 ônibus urbanos, ordenada pela condição de operação do sistema de refrigeração e nível sonoro emitido.

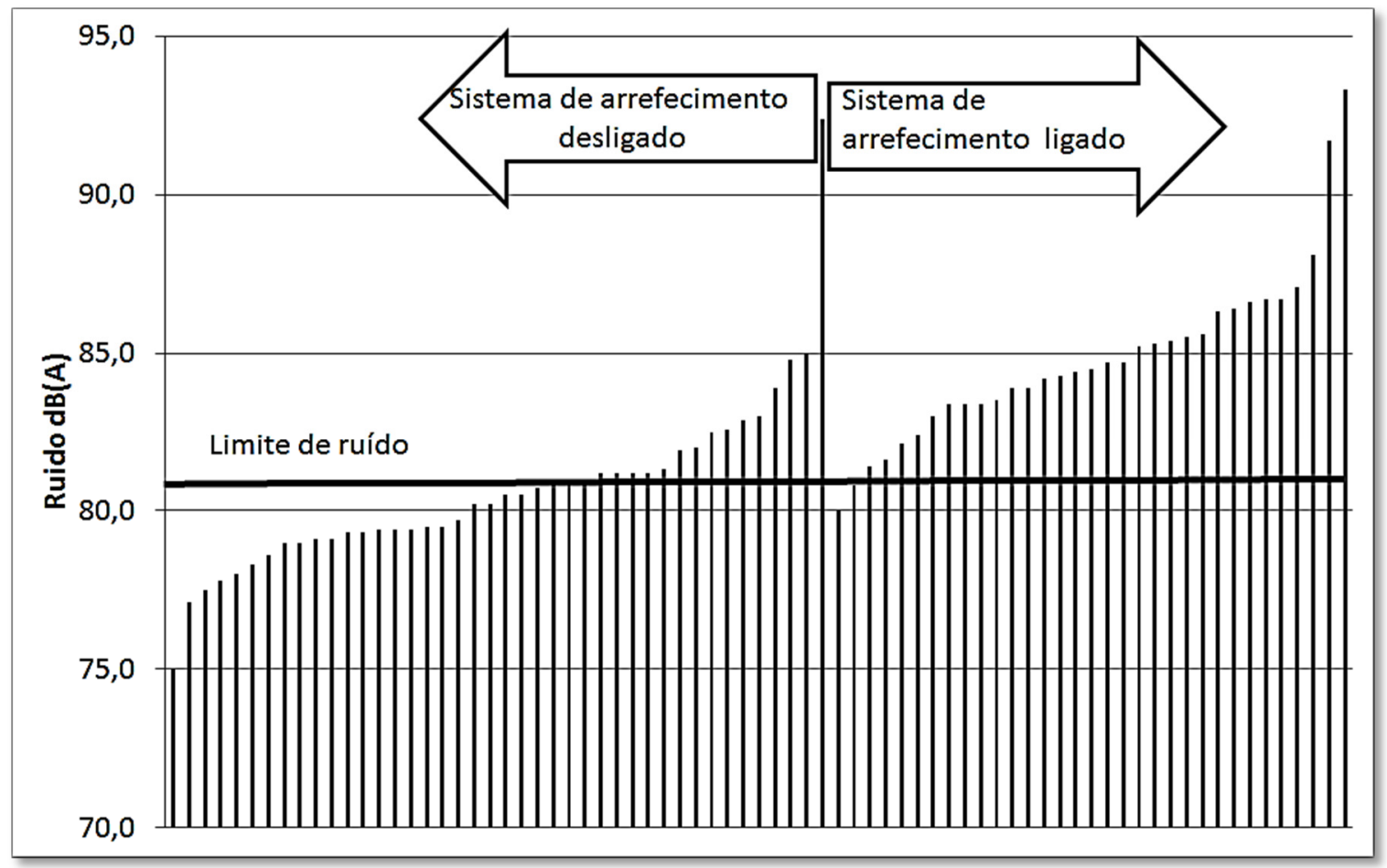

Fonte: dados próprios 
Deve-se considerar também que o encarroçamento de ônibus é muito diferente do de caminhões. Normalmente caminhões são equipados apenas com motores frontais, o que torna o arrefecimento mais fácil. Já os ônibus podem ser fabricados com motores frontais, no meio do chassi e traseiros, ademais, o chassi pode ser em monobloco, articulados ou biarticulados, o que torna a refrigeração do motor muito mais complexa. Além disso, é importante ter em mente que a frota de VP é usada geralmente mais de 12 horas por dia e com uma quilometragem/ano cerca de três vezes maior que um VLP [17].

Ainda no processo de avaliação das condições reais de tráfego urbano, foi feita uma medição do ruído ambiental equivalente (Leq) nas ruas Paes Leme e Teodoro Sampaio, em São Paulo, que encontrou correspondência com estudo detalhado elaborado por VIANNA [18] e apontou valores bem acima dos $60 \mathrm{~dB}(\mathrm{~A})$ recomendados pela norma ABNT NBR 10151 [19]. Os ônibus foram os veículos com maior nível de Leq e os que mais excederam a $80 \mathrm{~dB}(\mathrm{~A})$ (Tabela 1).

\begin{tabular}{|c|c|c|}
\hline & Leq - dB(A) & $\begin{array}{c}\text { Volume de tráfego } \\
\quad>80 \mathrm{~dB}(\mathrm{~A})\end{array}$ \\
\hline Ônibus & 77,5 & $31 \%$ \\
\hline Motos & 75,5 & $16 \%$ \\
\hline Automóveis & 72,6 & $2 \%$ \\
\hline Leq Geral & 73,8 & \\
\hline Leq (VIANNA) & 74,7 & \\
\hline
\end{tabular}

E por fim, deve-se observar que os caminhões têm uma aplicação muito diferente dos ônibus urbanos. Mesmo que se compare com caminhões pequenos, utilizados geralmente para entregas nas cidades, a aplicação é diferente. Os ônibus têm uma forte característica de parar e arrancar, muito mais frequente e acentuada do que os caminhões que fazem entregas urbanas. Considerando que a regulamentação europeia é a mesma para ambas as aplicações, implica em um desenvolvimento único de veículos para aplicações diferentes, que não combina com a aplicação real do veículo, que não é eficaz e não há redução de custos por esta padronização.

\section{CONCLUSÕES}

Não há dúvidas que a norma ISO 362-1:2007 representa um avanço no controle de ruído de veículos, e que este procedimento pode ser seguido por muitos países ao redor do mundo, como desejam os fabricantes de veículos europeus, e permitir que os custos de desenvolvimento possam ser reduzidos. Mas, apesar dos esforços dos especialistas, pode-se observar que algumas omissões feitas no passado estão sendo mantidas, o que pode fazer com que o método $B$ tenha um sucesso parcial. 
Para garantir o sucesso do controle de ruído, é importante rever o trabalho feito até o momento e acrescentar os seguintes temas:

- Inspeção e manutenção de veículos usados;

- Controle da produção;

- O ruído do sistema de arrefecimento do motor;

- O ruído elevado dos VP em tráfego urbano

- Método de homologação adequado para cada aplicação.

Algumas modificações nos veículos usados podem ser feitas ao longo de sua vida, como a remoção de revestimentos para isolamento de ruído, mudanças no corpo do veículo, alterações nas configurações do sistema de escapamento e assim por diante. Por isso, é muito importante que os materiais utilizados no isolamento de ruído do veículo sejam verificados nas inspeções periódicas, especialmente para VP, a fim de manter o ruído dentro dos valores esperados.

Considerando que o processo de homologação é baseado em uma avaliação de protótipo, é necessário um controle efetivo da produção (CoP), a fim de garantir que todos os componentes utilizados para reduzir a emissão de ruído serão montados nos veículos durante a vida do modelo. Além disso, o programa de CoP deve garantir a sua qualidade durante toda a vida do modelo do veículo.

Considerando-se que o sistema de arrefecimento do motor em VP pode multiplicar por quatro a energia sonora liberada, não é possível continuar a negligenciar esta significativa fonte de ruído, pois a população está sendo exposta diariamente, o que compromete o bem estar público.

Com o conhecimento adquirido sobre as propriedades dos materiais e sobre aerodinâmica, é possível obter um bom isolamento de ruído, sem impacto significativo nos custos finais dos veículos, utilizando materiais baratos ou simplesmente modificando o formato da hélice, sem comprometer a eficácia do sistema de arrefecimento.

Considerando-se a alta proporção de VP em circulação que excedem os $80 \mathrm{~dB}(\mathrm{~A})$ e que a principal fonte de ruído dos VP é o sistema motriz, são necessários estudos mais profundos para se identificar outras causas-raiz de emissão de ruído além do sistema de arrefecimento, tais como retirada de material fono absorvente, degradação dos veículos, etc. e propor ações complementares para o enfrentamento deste problema. Possivelmente a solução passará por rever conceitos construtivos, procedimentos de testes de homologação e limites legais de ruído.

Como os ônibus urbanos têm aplicação muito diferente dos caminhões, um procedimento de teste específico deve ser desenvolvido para cada aplicação, de modo que um teste específico de ruído para os ônibus urbanos, próximo ao parar e arrancar, deve ser considerado. Os custos de desenvolvimento serão os mesmos, no entanto, a eficácia será maior.

Muitos países ao redor do mundo estão enfrentando o mesmo problema ambiental que a Europa sobre o ruído dos veículos e precisam melhorar a sua legislação, mas alguns especialistas não estão totalmente convencidos sobre os benefícios 
ambientais deste novo método, e tendem a adotar outros métodos, considerados mais eficazes, o que pode elevar o custo final dos veículos. Mas, se estas proposições forem consideradas, poder-se-á melhorar a regulamentação europeia, diminuindo a exposição ao ruído dos europeus e fazendo com que ela seja mais facilmente adotada por outros países.

\section{REFERÊNCIAS}

[1] Regulation (EU) No 540/2014 of the European Parliament and of the Council of 16 April 2014 on the sound level of motor vehicles and of replacement silencing systems, and amending Directive 2007/46/EC and repealing Directive 70/157/EEC.

[2] BRÜEL \& KJAER. Environmental Noise. Disponível em: http://www.bksv.com/Library/Primers, acesso em 02/04/2015.

[3] WORLD HEALTH ORGANIZATION (WHO). Burden of disease from environmental noise: Quantification of healthy life years lost in Europe. WHO Regional Office for Europe: Copenhagen, p. 126, 2011.

[4] INTERNATIONAL ORGANIZATION OF MOTOR VEHICLE MANUFACTURES (OICA). Development of a New Global Type Approval Test Procedure for Motor Vehicles With Regard to Their Noise Emissions. International Symposium of Automotive Engineering. Automotive Engineering Association, Symposium proceedings, 2007.

[5] SANDBERG, Ulf et alli. Noise emissions of road vehicles effect of regulations - final report 01-1. Linköping: International Institute of Noise Control Engineering, 2001.56 p.

[6] BRAUN et alli. Noise source characteristics in the ISO 362 vehicle pass-by noise test: literature review. Applied Acoustics, 74 (2013), pp.1241-1265, 2013.

[7] Diretiva 70/157/CEE do Conselho de 6 de fevereiro de 1970 relativa a aproximação das legislações dos estados-membros respeitantes ao nível sonoro admissível e ao dispositivo de escape dos veículos a motor.

[8] UNITED NATIONS ECONOMIC COMMISSION FOR EUROPE (UNECE). Regulation No. 51: Uniform provisions concerning the approval of motor vehicles having at least four wheels with regard to their noise emissions. E/ECE/324/Rev.1/Add.50/Rev.2-E/ECE/TRANS/505/Rev.1/Add.50/Rev.2, 2011.

[9] INTERNATIONAL STANDARD ORGANIZATION (ISO). ISO 362: Measurement of noise emitted by accelerating road vehicles engineering method - Part 1: $\mathbf{M}$ and $\mathbf{N}$ categories. London: British Standards Institution; 2007.

[10] INTERNATIONAL STANDARD ORGANIZATION (ISO). ISO 362: Measurement of noise emitted by accelerating road vehicles engineering method - Part 1: $\mathbf{M}$ and $\mathbf{N}$ categories. London: British Standards Institution; 2015.

[11] MOORE, D. B. The Revised ISO 362 Standard for Vehicle Exterior Noise Measurement. Sound and vibration, pp. 19-23, 2006.

[12] KEULEN, I. W. V., DUŠKOV, i. M. Inventory study of basic knowledge on tyre/road noise. Ministery of transportation and waterworks: Rijkswaterstaat, p. 106, 2005. 
[13] NATIONAL COOPERATIVE HIGHWAY RESEARCH PROGRAM (NCHRP). Acoustic Beamforming: Mapping Sources of Truck Noise.

Transportation Research Board: Washington, D. C., p. 80, 2009.

[14] EUROPEAN COMMISSION. Final Report SI2.408210 Tyre/Road Noise: Volume 2, 2001.

[15] ROOVERS, M.S., BLOKLAND, G.J. Literature study on the Rolling Noise of Truck Tyres. Ministery of transportation and waterworks: Rijkswaterstaat, p. 92, 2002.

[16] BOSCH. Automotive Handbook. 7th ed. Society of Automotive Engineers. Warrendale. 2007.

[17] BRUNI, Antônio Carlos e BALES, Marcelo. Curvas de intensidade de uso por tipo de veículo automotor da frota da cidade de São Paulo. CETESB, São Paulo: 2013. 68 p.

[18] VIANNA, Karina Mary de Paiva. Poluição sonora no município de São Paulo: avaliação do ruído e do impacto da exposição na saúde da população. Tese de Doutorado. Faculdade de Saúde Pública da Universidade de São Paulo - FSP-USP: São Paulo, 2014, 145 p.

[19] ABNT - Associação Brasileira de Normas Técnicas. Norma Técnica NBR 10151 - Acústica - Avaliação do ruído em área habitadas, visando o conforto da comunidade - Procedimento. ABNT: Rio de Janeiro, 2000. 


\section{ANEXO \\ CATEGORIAS DE VEÍCULOS DE ACORDO COM A ISO 362-1:2015}

\section{Categoria L}

Os veículos a motor com menos de quatro rodas Nota 1 da entrada: Comissão Económica das Nações Unidas para a Europa (UNECE) documento TRANS / WP.29 / 78 / Rev.1 / Amend.4 (26 de Abril 2005) estendeu a categoria $L$ de quatro rodas veículos, conforme definido pelo L6 e L7.

Categoria M

Veículos a motor com pelo menos quatro rodas e utilizados para o transporte de passageiros.

Categoria M1

Veículos destinados ao transporte de passageiros e que não mais de oito lugares sentados além do lugar do condutor.

Categoria M2

Veículos utilizados para o transporte de passageiros e com mais de oito lugares sentados além do lugar do condutor e com uma massa máxima não superior a 5000 kg. Nota 1 a entrada: Nesta definição, "massa máxima" é equivalente a "massa máxima autorizada" usado em outro lugar nesta parte do ISO 362.

Categoria M3

Veículos utilizados para o transporte de passageiros e com mais de oito lugares sentados além do lugar do condutor e com uma massa máxima superior a $5000 \mathrm{~kg}$. Nota 1 de entrada: Nesta definição, "massa máxima" é equivalente a "massa máxima autorizada" usado em outro lugar nesta parte do ISO 362.

Categoria $\mathrm{N}$

Veículos a motor com pelo menos quatro rodas e utilizado para o transporte de mercadorias.

Categoria N1

Veículos utilizados para o transporte de mercadorias com massa máxima autorizada não superior a $3500 \mathrm{~kg}$.

Categoria N2

Os veículos utilizados para o transporte de mercadorias com massa máxima autorizada superior a $3500 \mathrm{~kg}$, mas não superior a $12000 \mathrm{~kg}$.

Categoria N3

Veículos utilizados para o transporte de mercadorias com massa máxima autorizada superior a $12000 \mathrm{~kg}$. 